\title{
A Uniqueness Theorem for Riemann Problems
}

\author{
J. A. Smoller \\ Communicated by L. CESARI
}

1.

In a recent paper [5] we considered the problem of finding a solution to the Riemann problem with arbitrary jump data for an extended class of $2 \times 2$ quasilinear hyperbolic systems of equations, in the class $\mathscr{L}$ of solutions consisting of constant states separated by centered rarefaction and shock waves. It is the purpose of this note to obtain a uniqueness theorem for this problem. In order to obtain such a result, it is well-known [3] that one must impose additional conditions on the class of solutions in order to exclude certain extraneous unstable solutions. LAX [3] presented a stability condition on shocks, hereafter referred to as condition $(\mathscr{S})$, which presumably should imply uniqueness. This condition states that each point of a shock consists of the intersection of characteristics from precisely one characteristic family. We shall show here that this condition does indeed imply uniqueness in the class $\mathscr{L}$. We note that if by a shock we mean a discontinuity satisfying the jump conditions (Rankine-Hugoniot conditions) which also comes from the intersection of characteristics (not necessarily from one family), then it was shown in [1] that a necessary condition for uniqueness is that each point of a shock comes from exactly one characteristic family. Thus our result shows that this condition is also sufficient for uniqueness. We remark that in the papers $[2,4]$ uniqueness theorems for Riemann problems are proved for some very restrictive hyperbolic systems.

\section{2.}

We consider the hyperbolic system of equations

$$
u_{t}+f(u, v)_{x}=0, \quad v_{t}+g(u, v)_{x}=0,
$$

and we let $F=(f, g)$. We assume throughout this paper that $f_{v}<0$ and $g_{u}<0$, so that $d F$ has real and distinct eigenvalues $\lambda_{2}(u, v)>\lambda_{1}(u, v)$, with left and right eigenvectors $l_{i}$ and $r_{i}, i=1,2$, and, in addition, we assume that $l_{i} d^{2} F\left(r_{j}, r_{j}\right)>0$, $i, j=1,2$, and $l_{i} r_{i}>0, i=1,2$. These conditions were studied in $[1,5]$ and imply a certain convexity and monotonicity of the shock and wave curves.

We shall study the Riemann problem for (1), so that we consider solutions of (1) which satisfy

$$
(u(0, x), v(0, x))=\left\{\begin{array}{l}
\left(u_{l}, v_{l}\right), x<0 \\
\left(u_{r}, v_{r}\right), x>0
\end{array}\right.
$$


for arbitrary preassigned constant vectors $\left(u_{l}, v_{l}\right)$ and $\left(u_{r}, v_{r}\right)$. By a solution of the problem (1)-(2) we mean a vector function $U=(u, v)$ which consists of (at most) three constant states separated by centered shocks and rarefaction waves of either the first or second type (characteristic family), which satisfies (1) in the classical sense in the regions where there is no shock (discontinuity), satisfies the RankineHugoniot condition across shocks, and satisfies the initial data. We shall also assume that one of the following stability conditions holds across the shocks $x=x(t)$ :

$$
\begin{array}{ll}
\lambda_{1}(U(t, x+0))<\sigma<\lambda_{1}(U(t, x-0)), & \sigma<\lambda_{2}(U(t, x+0)) \\
\lambda_{2}(U(t, x+0))<\sigma<\lambda_{2}(U(t, x-0)), & \sigma>\lambda_{1}(U(t, x-0)),
\end{array}
$$

where $\sigma=\dot{x}(t)$ is the shock speed. With this condition, it is easy to see that the following lemma holds:

Lemma 1. A solution of (1)-(2) can contain at most one shock or rarefaction wave of each type.

Fix $U_{l}=\left(u_{l}, v_{l}\right)$ and consider the states $(u, v)$ which can be connected to $U_{l}$ by a shock wave on the right of either type. These states satisfy the RankineHugoniot condition

$$
\left(u-u_{l}\right)\left(g(u, v)-g\left(u_{l}, v_{l}\right)\right)=\left(v-v_{l}\right)\left(f(u, v)-f\left(u_{l}, v_{l}\right)\right)
$$

and one of the conditions $(\mathscr{P})$. As in [5], our hypotheses imply that there exist two branches of (3), called the shock curves (of the first and second type), $v=$ $s_{1}\left(u ; U_{l}\right), u \geqq u_{l}$ and $v=s_{2}\left(u ; U_{l}\right), u \leqq u_{l}$, which are increasing convex downward and decreasing convex upward curves, respectively. Let $R$ and $S$ be the classical Riemann invariants of the system satisfying the non-linear system of equations $R_{t}+\lambda_{1} R_{x}=0, S_{t}+\lambda_{2} S_{x}=0$. We note that from the results of [5], the curves $R(u, v)=$ const. and $S(u, v)=$ const. are increasing convex downward and decreasing convex upward, respectively. If we let $\mathscr{A}$ denote the class of solutions of (1)-(2) satisfying ( $\mathscr{S})$ across shocks, we can state our main theorem as follows.

Theorem 1. There is at most one solution of the Riemann problem (1)-(2) in the class $\mathscr{A} \cap \mathscr{L}$.

The proof will be broken up into a series of lemmas, the first of which is

Lemma 2. The set of points different from $\left(u_{l}, v_{l}\right)$ satisfying (3) and (S) forms a one-manifold.

Proof. The points $(u, v)$ satisfying (3) can be written in the form $\phi(u, v$; $\left.u_{l}, v_{l}\right)=0$, and also can be written in the form $\sigma\left(u-u_{l}\right)=f(u, v)-f\left(u_{l}, v_{l}\right)$, $\sigma\left(v-v_{l}\right)=g(u, v)-g\left(u_{l}, v_{l}\right)$. If $\phi_{u}=\phi_{v}=0$ at some $(u, v) \neq\left(u_{l}, v_{l}\right)$, then at this point we have

$$
\begin{aligned}
& \left(u-u_{l}\right) g_{u}+\left(v-v_{l}\right)\left(\sigma-f_{u}\right)=0 \\
& \left(u-u_{l}\right)\left(\sigma-g_{v}\right)+\left(v-v_{l}\right) f_{v}=0
\end{aligned}
$$

so that $g_{u} f_{v}=\left(\sigma-f_{u}\right)\left(\sigma-g_{v}\right)$. But this is precisely the equation $\operatorname{det}[d F(u, v)-\sigma \eta]=0$ so that $\sigma=\lambda_{1}$ or $\sigma=\lambda_{2}$, and this contradicts $(\mathscr{S})$. Hence $\phi_{u}$ and $\phi_{v}$ can both vanish only at $\left(u_{l}, v_{l}\right)$, and this implies the lemma. 
Lemma 3. Suppose that $\bar{U}=(\bar{u}, \bar{v})$ satisfies (3) and one of the conditions $(\mathscr{S})$. Then $\bar{U}$ lies on one of the shock curves.

Proof. It was shown in [1] that for $(u, v)$ on the shock curve of the second type

$$
a_{2}(u, v)>h_{2}(u, v)>\left(v-v_{l}\right) /\left(u-u_{l}\right)
$$

where $a_{2}$ is the slope of the curve $R=R(u, v)$, and $h_{2}$ is the slope of the shock $v=s_{2}\left(u ; U_{l}\right)$. This implies that the curve $R=R\left(U_{l}\right)$ lies entirely above the shock curve $v=s_{2}\left(u ; U_{l}\right)$. For, the curves $R=$ const. starting on the shock curve $v=s_{2}(u$; $U_{l}$ ) all start out breaking to the right of the shock curve for increasing $u$. If any of these curves ever came back to the shock curve this would imply a singularity in the vector field. Similarly, it is not too hard to show for $(u, v)$ on the shock curve of the first type that $h_{1}(u, v)>a_{1}(u, v)>\left(v-v_{l}\right) /\left(u-u_{l}\right)$. Thus the curve $S=S\left(U_{l}\right)$ lies entirely above the shock curve $v=s_{1}\left(u ; U_{l}\right)$. This situation is illustrated in Fig. 1.

We now consider the states which can be connected to $U_{l}$ by an $i$-shock on the left. An analysis similar to that above shows that the standard branch of this manifold, i.e. the "shock curve" (the component connected to $\left.U_{l}\right) v=s_{i}^{\prime}(u$; $\left.U_{l}\right), i=1,2$, lies between the curves $S=S\left(U_{l}\right)$ and $R=R\left(U_{l}\right)$. Moreover, $s_{1}^{\prime}\left(u ; U_{l}\right)$ is increasing convex downward and $v=s_{2}^{\prime}\left(u, U_{l}\right)$ is decreasing convex upward.

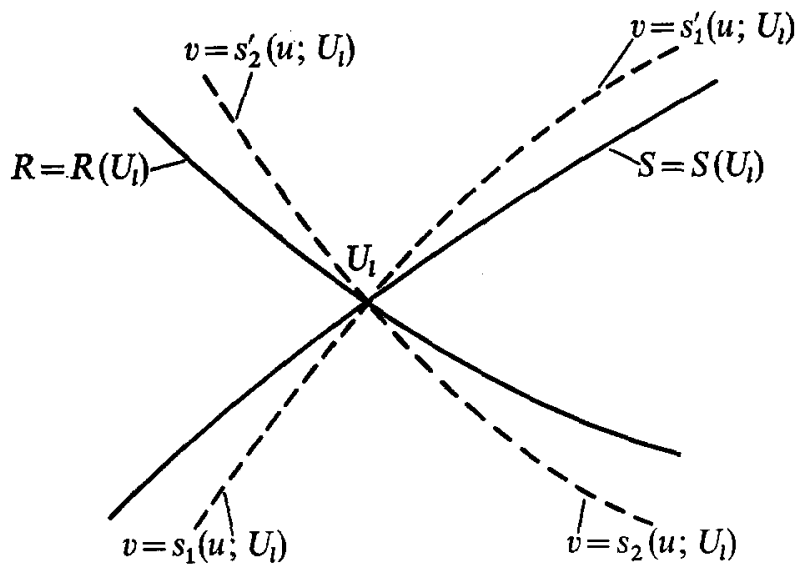

Fig. 1

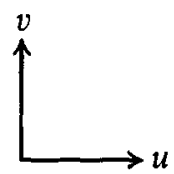

Suppose now that $\bar{U}$ satisfies one of the conditions $(\mathscr{S})$, say the second (the case where $\bar{U}$ satisfies the first condition is similar). First suppose that $\bar{U}$ lies in the region below $v_{l}$ between or on the curves $R=R\left(U_{l}\right)$ and $S=S\left(U_{l}\right)$. We claim that if $\bar{U}$ is not on the shock curve $v=s_{2}\left(u ; U_{l}\right)$ then $\bar{u}<u_{l}$. To see this, first note that from Lemma $3, \bar{U}$ is not an isolated point, and that if $\bar{u} \geqq u_{l}$, then all the points which satisfy (3) and the second condition $(\mathscr{S})$ also satisfy $d v / d u=h_{2}(u, v)$ with $h_{2}<0$, (cf. [1]). Thus we see that the branch of (3) containing $\bar{U}$ either cuts $v=s_{2}\left(u ; U_{l}\right)$ or it cuts $v=v_{l}$ or it cuts $u=u_{l}$. If it cuts $v=s_{2}\left(u ; U_{l}\right)$ we have a contradiction. Suppose that it cuts $u=u_{l}$, say at $\left(u_{l}, v_{l}^{\prime}\right)$. Then since $\left(u_{l}, v_{l}^{\prime}\right)$ 
satisfies (3) we have

$$
0=\left(v-v_{l}\right)\left(f\left(u_{l}, v^{\prime}\right)-f\left(u_{l}, v_{l}\right)\right)=\left(v^{\prime}-v_{l}\right)^{2} f_{v}\left(u_{l}, \xi\right)
$$

for some $\xi$ so that $v^{\prime}=v_{l}$ since $f_{v} \neq 0$. Thus this branch goes through $\left(u_{l}, v_{l}\right)$. This is impossible unless this branch is the same as the usual branch of the shock curve (cf. [1], equation (2.11) ff.). In a similar way, we can treat the case when the branch of (3) containing $\bar{U}$ intersects $v=v_{l}$.

Thus we may assume $\bar{u}<u_{l}$. If now $\bar{U}$ were to the right of $v=s_{1}\left(u ; U_{l}\right)$, then from the results of [5] there is a point $U$ on $v=s_{1}\left(u, U_{l}\right)$ such that $U$ and $\bar{U}$ are connected by a 2-shock. Hence we have

$$
\begin{aligned}
& \sigma_{1}[U-\bar{U}]=F(U)-F(\bar{U}) \\
& \sigma_{2}\left[U_{l}-U\right]=F\left(U_{l}\right)-F(U) \\
& \sigma_{3}\left[U_{l}-\bar{U}\right]=F\left(U_{l}\right)-F(\bar{U}),
\end{aligned}
$$

so that

$\sigma_{3}\left[U_{l}-U\right]+\sigma_{3}[U-\bar{U}]=F\left(U_{l}\right)-F(U)+F(U)-F(\bar{U})=\sigma_{2}\left[U_{l}-U\right]+\sigma_{1}[U-\bar{U}]$

and thus

$$
\left(\sigma_{3}-\sigma_{2}\right)\left[U_{t}-U\right]+\left(\sigma_{3}-\sigma_{1}\right)[U-\bar{U}]=0
$$

This implies that $\sigma_{1}=\sigma_{2}=\sigma_{3}$. However, condition $(\mathscr{S})$ implies that $\sigma_{3}>\lambda_{1}\left(U_{l}\right)$ and $\lambda_{1}\left(U_{l}\right)>\sigma_{2}$ must hold. This is a contradiction, so that $\bar{U}$ must lie to the left of the curve $v=s_{1}\left(u ; U_{l}\right)$. In this case, we consider the curve $v=s_{2}(u ; \bar{U})$. This curve must intersect the shock curve $v=s_{1}\left(u ; U_{l}\right)$, say at $U$. Then we have equalities of the form (5) holding again. Hence, as before, $\sigma_{1}=\sigma_{2}=\sigma_{3}$. But using ( $\left.\mathscr{S}\right)$, again we have $\sigma_{3}>\lambda_{1}\left(U_{l}\right)$ and $\lambda_{1}\left(U_{l}\right)>\sigma_{2}$. Thus $\bar{U}$ must lie on the curve $v=$ $s_{1}\left(u ; U_{l}\right)$.

Next, suppose that $\bar{U}$ were in the region between (and not on) the curves $S=S\left(U_{l}\right), R=R\left(U_{l}\right)$ with $\bar{u}>u_{l}$. We shall show that this is impossible. To do this, we first construct the curve $v=s_{1}(u ; \bar{U})$ and note that this curve cannot intersect $S=S\left(U_{l}\right)$ (otherwise $S=S(\bar{U})$ intersects this curve), so that it intersects $v=s_{2}\left(u ; U_{l}\right)$, say at $U$. We than have equalities of the form (5) holding again. Hence, as before $\sigma_{1}=\sigma_{2}=\sigma_{3}$, but then since $\lambda_{2}(\bar{U})>\lambda_{1}(\bar{U})>\sigma_{2}=\sigma_{1}>\lambda_{2}(\bar{U})$, we obtain the desired contradiction. If now $\bar{U}$ were in the region between (and not on) the curves $S=S\left(U_{l}\right), R=R\left(U_{l}\right)$, we would first construct the curve $v=s_{2}(u ; \bar{U})$ and obtain a similar contradiction.

Finally, suppose that $\bar{U}$ is in the region above $v_{l}$, between or on the curves $S=S\left(U_{l}\right), R=R\left(U_{l}\right)$. If $\bar{U}$ were on the curve $v=s_{2}^{\prime}\left(u ; U_{l}\right)$, then we would have $\lambda_{2}(\bar{U})>\lambda_{2}\left(U_{l}\right)>\lambda_{2}(\bar{U})$, an impossibility. If $\bar{U}$ were on the curve $v=s_{1}^{\prime}\left(u ; U_{l}\right)$, then we would have $\sigma\left[U_{l}-\bar{U}\right]=F\left(U_{l}\right)-F(\bar{U})$ and $\sigma^{\prime}\left[U_{l}-\bar{U}\right]=F\left(U_{l}\right)-F(\bar{U})$, whence $\sigma=\sigma^{\prime}$. But then $\lambda_{2}(\bar{U})>\sigma^{\prime}=\sigma>\lambda_{2}(\bar{U})$, which is again impossible. Now suppose $\bar{U}$ were in the region between or on $S=S\left(U_{l}\right)$ and $v=s_{1}^{\prime}\left(u ; U_{l}\right)$. We construct the curve $v=s_{1}(u ; \bar{U})$ and note that it meets $v=s_{1}^{\prime}\left(u ; U_{l}\right)$, say at $U$. We again have relations of the form (5) holding so that we again get $\sigma_{1}=\sigma_{2}=\sigma_{3}$. But then we get the contradiction $\lambda_{2}(U)>\sigma_{1}=\sigma_{3}>\lambda_{2}(U)$. Similarly if $\bar{U}$ lies in the region between or on $R=R\left(U_{l}\right)$ and $v=s_{2}^{\prime}\left(u ; U_{l}\right)$, we construct the curve 
$v=s_{2}(u ; \bar{U})$, note that it meets $v=s_{2}^{\prime}\left(u ; U_{l}\right)$, and get a similar contradiction. If now $\bar{U}$ lies between the curves $v=s_{1}^{\prime}\left(u ; U_{l}\right)$, and $v=s_{2}^{\prime}\left(u ; U_{l}\right)$, we construct the curve $v=s_{2}(u ; \bar{U})$, note that it meets $v=s_{1}^{\prime}\left(u ; U_{l}\right)$ at $U$, and get a similar contradiction $\lambda_{2}(\bar{U})>\sigma>\lambda_{2}(\bar{U})$. The proof of the lemma is complete.

Let $\mathscr{U}$ be any set of points in the $(u-v)$ plane. We define

$$
\begin{aligned}
& P_{1}(\mathscr{U})=\left\{(u, v): v=w_{1}\left(u ; U_{0}\right), U_{0}=\left(u_{0}, v_{0}\right) \in \mathscr{U}, u \geqq u_{0}\right\} \\
& P_{2}(\mathscr{U})=\left\{(u, v): v=w_{2}\left(u ; U_{0}\right), U_{0}=\left(u_{0}, v_{0}\right) \in \mathscr{U}, u \leqq u_{0}\right\} \\
& \Sigma_{i}(\mathscr{U})=\left\{(u, v): v=s_{i}(u, U), U \in \mathscr{U}\right\}, \quad i=1,2 .
\end{aligned}
$$

Now fix a point $U_{l}$ in the $u-v$ plane, and let $\rho_{i}=P_{i}\left(U_{l}\right), \sigma_{i}=\Sigma_{i}\left(U_{l}\right), i=1,2$.

Lemma 4. Outside of the curve $\rho_{1} \cup \sigma_{1}$, the set $P_{2}\left(\rho_{1} \cup \sigma_{1}\right) \cup \Sigma_{2}\left(\rho_{1} \cup \sigma_{1}\right)$ is a disjoint union of curves.

Proof. The elements of the set in question consist of the shock and rarefaction wave curves of the second type, originating on the curve $\rho_{1} \cup \sigma_{1}$ (which is itself the shock and wave curve of the first type originating on $\left.U_{l}\right)$. As was shown in [5], the set $\rho_{1} \cup \rho_{2} \cup \sigma_{1} \cup \sigma_{2}$ divides the $(u-v)$ plane into four regions I, II, III, IV, as the following diagram shows:

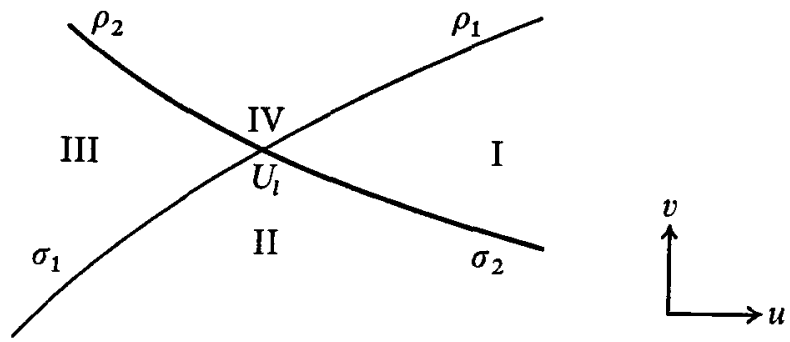

Fig. 2

Suppose now that there exist $U_{1}=\left(u_{1}, v_{1}\right)$ and $U_{2}=\left(u_{2}, v_{2}\right), u_{2}>u_{1}$, on $\rho_{1} \cup \sigma_{1}$ such that there is a point $\bar{U}$ in $\left\{P_{1}\left(U_{1}\right) \cup \Sigma_{1}\left(U_{1}\right)\right\} \cap\left\{P_{1}\left(U_{2}\right) \cup \Sigma_{1}\left(U_{2}\right)\right\}$. We will show that this is impossible and this will complete the proof. The uniqueness theorem for ordinary differential equations shows that $\bar{U}$ cannot lie in regions III or IV. Moreover, it follows from Theorem 2.9 of [1] that under the assumption $(\mathscr{S}), \bar{U}$ cannot lie in region $I$. Thus we must only consider the case where $\bar{U}$ is in region II. Here $\bar{U}$ lies on $\Sigma_{2}\left(U_{1}\right) \cap \Sigma_{2}\left(U_{2}\right)$, and we shall first consider the case where $u_{2} \neq u_{l}$. We construct the curve $S=S\left(U_{l}\right)$ for $u \leqq u_{l}$. Then there are points $U_{3}, U_{4}$ on this curve such that $U_{2} \varepsilon \Sigma_{2}\left(U_{3}\right)$ and $\Sigma_{2}\left(U_{4}\right) \cap \Sigma_{2}\left(U_{3}\right)=\bar{U}$ (cf. [1], Lemma 2.7). Hence $\Sigma_{2}\left(U_{3}\right) \cap \Sigma_{2}\left(U_{4}\right) \neq \phi$, and this contradicts Theorem 2.9 of [1].

Now consider the case where $u_{2}=u_{l}$. Choose a point $U_{3}=\left(u_{3}, v_{3}\right)$ with $u_{3}>u_{l}$ such that $U_{l} \varepsilon \Sigma_{1}\left(U_{3}\right)^{\star}$. Then, as before, points $(u, v) \varepsilon \Sigma_{1}\left(U_{3}\right)$ lie below $\Sigma_{1}\left(U_{l}\right)$ for

$\star$ If $U_{l}$ is any point in the $(u-v)$ plane and $u_{1}>u_{l}, u_{1}$ close to $u_{l}$, then the mapping from the line $u=u_{1}$ into the line $u=u_{l}$, given by $\left(u_{1}, v\right) \rightarrow \Sigma_{1}(u, v) \cap\left\{u=u_{l}\right\}$, is continuous since the shock curves have positive slopes. The point $\left(u_{1}, v_{l}\right)$ maps below $U_{l}$, and since the slopes of the shock curves are continuous functions, there is a $\bar{v}$ such that $\left(u_{1},\right) \bar{v}$ maps above $U_{l}$. Thus there is a point $\left(u_{1}, v\right)$ mapping onto $U_{l}$. 
$u<u_{l}$. Let $U_{4}$ be any point on $\Sigma_{1}\left(U_{l}\right)$ between $U_{l}$ and $U_{2}$. Then $\Sigma_{2}\left(U_{4}\right) \cap \Sigma_{1}\left(U_{3}\right) \neq \phi$, $\Sigma_{2}\left(U_{4}\right) \cap \Sigma_{2}\left(U_{l}\right) \neq \phi$, and if $U_{5}$ lies in this first intersection we see that $\Sigma_{2}\left(U_{5}\right) \cap \Sigma_{2}$ $\left(U_{l}\right) \neq \phi$; thus we have reduced this case to the previous one. This completes the proof of the lemma.

In view of Lemmas 1, 3 and 4, we see that the proof of Theorem 1 now follows.

In [1] it was proved that the second parts of both conditions $(\mathscr{S})$ are necessary conditions for uniqueness. Thus, if we define an $i$ shock, $i=1,2$, to be a discontinuity $x=x(t)$ satisfying only

$$
\lambda_{i}(U(t, x-0))>\sigma>\lambda_{i}(U(t, x+0)),
$$

where $\sigma=\dot{x}(t)$ is the shock speed, we can state

Theorem 2. There is a unique solution of (1)-(2) in the class $\mathscr{L}$ if and only if $\sigma<\lambda_{2}(U(t, x+0))$ across a shock of type 1 and $\sigma>\lambda_{1}(U(t, x-0))$ across a shock of type 2 .

Hence, if any of the conditions of Lemma 2.5 of [1] hold, for example if $\lambda_{2} \geqq 0 \geqq \lambda_{1}$, then there is at most one solution of (1)-(2) in $\mathscr{L}$. Finally, we remark that Theorem 1, together with the results of [5], solves the problem of existence and uniqueness of the solution of the Riemann problem for the system (1) in the class $\mathscr{L}$.

This research was supported in part by N.S.F. Grant \# 01048 .

\section{References}

1. Johnson, J. L., \& J. A. Smoller, Global solutions for an extended class of hyperbolic systems of conservation laws. Arch. Rational Mech. Anal., 32, 169-189 (1969).

2. Kuznecov, N. N., Problem of the disintegration of an arbitrary discontinuity for a system of quasi-linear equations of the first order. Doklady Akad. Nauk. 131, 503-506 (1960). Translated in Sov. Math. 1, 282-285 (1960).

3. Lax, P. D., Hyperbolic systems of conservation laws. II. Comm. Pure Appl. Math. 10, $537-566(1957)$.

4. RozDESTVENSKII, B. L., Discontinuous solutions of systems of quasi-linear hyperbolic equations. Usp. Mat. Nauk. 15, 59-117 (1960). Translated Russian Math. Surveys 15, 53-111 (1960).

5. SMOLLER, J. A., On the solution of the Riemann problem with general step data for an extended class of hyperbolic systems. Mich. Math. J., to appear.

Department of Mathematics University of Michigan

Ann Arbor 